\title{
Comparison of Genotoxic Damage in Monolayer Cell Cultures and Three-Dimensional Tissue-Like Cell Assemblies
}

E. Behravesh ${ }^{\mathrm{a}}$, K. Emami ${ }^{\mathrm{b}}, \mathrm{H} . \mathrm{Wu}^{\mathrm{c}}$, and S. Gonda ${ }^{\mathrm{d}}$

${ }^{2}$ Universities Space Research Association, Division of Space and Life Sciences. 3600 Bay Area Blvd, Houston, TX 77058, USA.

${ }^{\mathrm{b}}$ Wyle Laboratories, Inc., Life Sciences Systems and Services, 1290 Hercules Drive, Suite 120, Houston, Texas 77058, USA.

${ }^{c}$ National Aeronautical and Space Administration, Johnson Space Center, 2101 NASA Road One, Mail Code SK, Houston, Texas 77058, USA.

${ }^{\mathrm{d}}$ National Aeronautical and Space Administration, Johnson Space Center, 2101 NASA

Road One, Mail Code SJ, Houston, Texas 77058, USA.

Corresponding Author Permanent Address:

Steve R. Gonda

NASA Johnson Space Center

2101 NASA Road One

Mail Code: SJ

Houston, Texas 77058, USA

Phone: (281) 483-8745

Fax: (281) 483-0402

Email: sgonda@ems.jsc.nasa.gov 


\section{Abstract}

Assessing the biological risks associated with exposure to the high-energy charged particles encountered in space is essential for the success of long-term space exploration. Although prokaryotic and eukaryotic cell models developed in our laboratory and others have advanced our understanding of many aspects of genotoxicity, in vitro models are needed to assess the risk to humans from space radiation insults. Such models must be representative of the cellular interactions present in tissues and capable of quantifying genotoxic damage. Toward this overall goal, the objectives of this study were to examine the effect of the localized microenvironment of cells, cultured as either 2-dimensional (2D) monolayers or 3-dimensional (3D) aggregates, on the rate and type of genotoxic damage resulting from exposure to iron charged particles, a significant portion of space radiation. We used rodent transgenic cell lines containing 50-70 copies of a LacI transgene to provide the enhanced sensitivity required to quantify mutational frequency and type in the 1,100-bp LacI target as well as assessment of DNA damage to the entire 45-kbp construct. Cultured cells were exposed to high-energỹ iron charged particles at Brookhaven National Laboratory's Alternating Gradient Synchrotron facility for a total dose of $0,0.1,0.25,0.5,1.0$, or 2.0 Gy and allowed to recover for 0,1 , or 7 days, after which mutational type and frequency were evaluated. The mutational frequency was found to be higher in 3D samples than in 2D samples at all radiation doses. Mutational frequency also was higher at 7 days after irradiation than immediately after exposure. DNA sequencing of the mutant targets revealed that deletional mutations contributed an increasingly high percentage (up to $27 \%$ ) of all mutations in cells as the dose was 
increased from 0.5 to 2 Gy. Several mutants also showed large and complex deletions in multiple locations within the LacI target. However, no differences in mutational type were found between the $2 \mathrm{D}$ and the $3 \mathrm{D}$ samples. These $3 \mathrm{D}$ tissue-like model systems can reduce the uncertainty involved in extrapolating risk between in vitro cellular and in vivo models.

\section{Introduction}

Earth's atmosphere serves an important purpose in sustaining life by filtering most of the Sun's ultraviolet and space ionizing radiation (Lyon, 2000). Galactic cosmic rays and solar particle events contain high-energy, high-atomic-number energetic (HZE) ionizing particles that pose a significant health risk to human crews on space missions (Setlow, 1999). These HZE particles, of which iron is the most abundant, have great penetration power and a greater potential of inducing biological damage through ionizing radiation than do types of radiation found on Earth. The specific mutational frequency and mutational spectra, the type of damage at the DNA level, caused by HZE radiation are active areas of current research (Grosovsky et al., 2001, Kronenberg, 1994, Kronenberg and Little, 1989, Kronenberg et al., 1995, Chang et al., 2001). Such studies have typically used either traditional 2-dimensional (2D) cell cultures of adherent cell populations or animal models. The $2 \mathrm{D}$ in vitro systems do not allow assessment of the dynamic effects of intercellular interactions within tissues (Barcellos-Hoff and Brooks, 2001), whereas potentially confounding factors tend to be overlooked in animal models 
because of current gaps in knowledge of the differences in metabolism and genetic repair processes between species(Balmain and Harris, 2000, Vogel et al., 1996).

NASA has been a pioneer in the design of bioreactors for culture of 3dimensional (3D) tissue-like models (Pei et al., 2002, Gonda et al., 2001). The ability of bioreactors to support the development of multicellular, multilayer, 3D tissue-like constructs has been attributed to the low-shear environment, randomization of the gravity vector, and colocalization of cells, cell aggregates, and microcarrier beads within the bioreactor.

To assess late effects of HZE radiation, DNA mutagenesis seems to be an ideal biological endpoint because of the likelihood that it contributes to carcinogenesis and perhaps to clinical disease (McKinzie et al., 2001, Cairns, 2002). To identify mutagenesis in any model, specific targets need to be used to allow detection of the type of damage caused by the specific environmental stressor. Genetically engineered constructs are advantageous in that no selective pressures are associated with the target that might prevent the identification of mutations in native targets (Mirsalis, 1995, Kohler et al., 1991). Several transgenic animal models have been developed that contain multiple copies of a target gene for detecting mutations (Nohmi et al., 2000, Ikehata et al., 2003, Masumura et al., 2002). Rare sequence changes in populations of DNA molecules can be detected in these transgenic animals by analyzing the DNA target to investigate the induction of DNA mutations-potentially the first step in carcinogenesis.

One transgenic target makes use of the lac operon. The lacI gene encodes the lac repressor protein, which represses the lacZ gene (which in turn codes a portion of $\beta$ galactosidase). Mutations in the lacI gene that inactivate the lac repressor protein allow 
transcription of the lac $Z$ gene and production of $\beta$-galactosidase. Mutants producing $\beta$ galactosidase are detectable as blue plaques in the presence of X-gal whereas nonmutants are colorless. Both transgenic animals and cells containing the LacI target have been used to assess DNA damage induced by a variety of environmental stressors.

The objective of this study was to analyze the effect of the microenvironment of transgenic rodent cells, cultured either in 2D monolayers or as 3D tissue-like aggregates, on mutagenesis at a transgenic target after exposure to irradiation in the form of iron charged particles. Mutagenesis was also measured over time to assess the functionality of normal cellular processes involved in the repair of DNA. Mutagenesis was quantified in terms of mutational frequency and type.

\section{Materials and Methods}

\subsection{Cell Cultures}

The cell line used, Rat2 $\lambda$ fibroblasts (Stratagene, La Jolla, CA), has been genetically engineered to contain approximately 60 copies of the LacI gene per cell to facilitate quantification of mutations. Rat $2 \lambda$ cells were cultured in Dulbecco's modified Eagle medium (Invitrogen, Carlsbad, CA) containing 10\% fetal bovine serum (Sigma, St. Louis, MO), 1\% L-glutamine (Invitrogen), and 1\% penicillin/streptomycin (Invitrogen) in a $99 \%$ humidity, $5 \% \mathrm{CO}_{2}$ incubator at $37^{\circ} \mathrm{C}$. Cells were enzymatically lifted at the required times with trypsin-ethylenediamine tetraacetic acid (EDTA) (Invitrogen). 


\subsection{Three-Dimensional Cell Cultures}

Three-dimensional tissue-equivalent analogues were formed in a high aspect ratio vessel (HARV) bioreactor (Synthecon, Houston TX), which provides a low-shear environment. Each of eight $50 \mathrm{ml}$ vessels was inoculated with $5 \times 10^{5}$ Cytodex 3 beads (Sigma) and completely filled with medium. The bioreactors were then placed in an incubator and the vessels rotated at 10 revolutions per minute.

Cells were harvested with trypsin-EDTA before reaching confluence, centrifuged, and resuspended in growth medium. On day $0,10^{7}$ Rat $2 \lambda$ fibroblasts were inoculated into each HARV (to produce a cell-to-bead ratio of 20:1), and the HARVs were rotated at 12 revolutions per minute for up to 20 days. Bioreactors were monitored daily for cellular metabolites, $\mathrm{pH}$, dissolved gases $\left(\mathrm{pO}_{2}\right.$ and $\left.\mathrm{pCO}_{2}\right)$, and bicarbonate concentration. Cell attachment, growth, and formation of aggregates were monitored by phase contrast microscopy over the 20-day culture period.

\subsection{Quantifying Numbers of Cells Per Aggregate}

The numbers of cells per aggregate were quantified by using PicoGreen (Molecular Probes, Eugene, OR) double-stranded DNA fluorescent dye in 96-well plates according to the manufacturer's instructions. PicoGreen is a cell membrane permeable dye that fluoresces once bound specifically to double stranded DNA.

\subsection{Mutagen Treatments}

The mutagen ethylnitrosourea (ENU) was used as a positive control and exposed to cells based on published the literature (Zimmer et al., 1996). Briefly, 2D Rat2 $\lambda$ cell 
cultures were exposed to ENU at $0-1,000 \mu \mathrm{g} / \mathrm{ml}$ in serum-free medium for $10 \mathrm{~min}$, after which the cells were washed, cultured in medium containing serum for another 7 days, and preserved by snap freezing on dry ice and storage at $-80^{\circ} \mathrm{C}$ for mutational analysis.

\subsection{Radiation Treatments}

Aggregates were placed in 25-ml tissue culture module bags (American Fluoroseal, Gaithersburg, MD) made from gas-permeable polytetrafluoroethylene material to allow continuous cell culture. Approximately $10^{7}$ cells, cultured on beads that had formed aggregates, were placed inside each bag. Monolayer cultures and the bagged aggregates were then shipped to Brookhaven National Laboratory (Upton, NY).

For the 2D monolayer controls, fibroblasts were subcultured onto T-25 flasks at $50 \%$ confluence at 2 days before irradiation. The flasks and the bagged 3D aggregates were then exposed to heavy ion (iron) irradiation at an energy of $1 \mathrm{GeV} / \mathrm{amu}$ for a total dose of $0.1,0.25,0.5,1.0$, or $2.0 \mathrm{~Gy}$. The dose rate was $1 \mathrm{~Gy} / \mathrm{min}$ for all doses. Controls not exposed to irradiation were transported with the exposed samples. Samples were then either immediately washed twice with phosphate-buffered saline (PBS) and snap-frozen in dry ice or cultured for an additional 1 or 7 days, after which they were washed twice with PBS and snap-frozen in a dry ice/ethanol bath.

\subsection{DNA Isolation}

DNA from both the 2D monolayer cultures and the $3 \mathrm{D}$ aggregates was isolated with a MiniPrep DNA isolation kit (Promega, Madison, WI) according to the manufacturer's instructions. The precipitated DNA was dissolved in $25 \mu 1$ of Tris-EDTA 
buffer (10 mM Tris in 4 mM EDTA [pH 8]). DNA concentrations were determined by using PicoGreen dye (Molecular Probes) after the DNA solution had been diluted 1:1,000 in Tris-EDTA buffer.

\subsection{DNA Packaging}

The lambda transgenic shuttle vector containing the lacl target gene was recovered from the isolated DNA and packaged into viable lambda phage particles by using Transpack Packaging Extract (Stratagene). Assays were conducted according to the manufacturer's instructions (Zhang et al., 2002, Rogers et al., 1995, Provost et,al., 1993) except that the concentration of the bacteria was increased by a factor of 10 to reduce phage migration and the packaged DNA samples were first titrated to allow 30,000 plaque-forming units (PFU) to form on $25 \times 25 \mathrm{~cm}$ assay trays. In this assay, plaques with mutations are blue and non-mutated plaques are colorless.

\subsection{Mutant Isolation and Purification}

Blue (mutated) plaques were cored with a $200-\mu 1$ pipet tip and each resuspended in $500 \mu 1$ of SM buffer $\left(100 \mathrm{mM} \mathrm{NaCl}, 10 \mathrm{mM} \mathrm{MgSO}_{4}, 50 \mathrm{mM}\right.$ Tris [pH 7.5], and $0.01 \%$ gelatin; Fisher Scientific, Houston TX) with $50 \mu 1$ of chloroform (Sigma) and stored at $4^{\circ} \mathrm{C}$. Then $5 \mu 1$ from each of the solutions containing mutant plaques were replated in $10-$ mm petri dishes, a well isolated mutant cored, resuspended in SM-chloroform, and preserved at $4^{\circ} \mathrm{C}$.

\subsection{DNA Sequencing}


Mutations in lacI-bearing phage were determined by amplifying the region of interest with the following LacI primers: forward $(-41$ to -20$), 5^{\prime}-$ GAATGGTGCAAAACCTTTCGCG-3'; and reverse (1151 to 1128), 5'TGCCTAATGAGTGAGCTAACTCAC-3'. Primers encompassing the entire LacI target DNA were used to sequence the lacI gene.

\subsection{Statistical Analyses}

A full mixed factor factorial design was used, with culture conditions at two levels (either 3D aggregate or 2D monolayer), the time of culture after exposure at three levels $(0,1$, or 7 days $)$ and dose delivered at 6 levels $(0,0.1,0.25,0.5,1.0$, or $2.0 \mathrm{~Gy})$. Both main factor effects and interactions were tested. Microenvironment and dose were also tested as nominal factors to test the validity of this statistical model. The $\alpha$ level for significance was set at 0.05 .

\section{Results}

\subsection{Tissue Cultures}

Over the course of 20 days of continuous culture in the HARV bioreactors, the transgenic fibroblasts attached to the microcarrier beads and formed 3D aggregates consisting of approximately 20 Cytodex 3 beads that were completely engulfed by proliferating Rat $2 \lambda$ cells. Glucose and lactate levels during the 20-day cultures are shown 
in Figure 1. At least $25 \mathrm{ml}$ of medium was exchanged daily after the second day of culture to maintain the metabolite levels within an acceptable range.

\subsection{Positive Control}

The mutational frequency and packaging efficiency of Rat $2 \lambda$ cells after exposure to ENU are shown in Figure 2. A near-linear trend of increasing mutational frequency was noted with increasing dose (Figure 2A), but packaging efficiency, normalized to the untreated control, was not related to mutational frequency (Figure 2B).

\subsection{Mutational Frequency}

To quantify mutational frequencies, on average, a total of 200,000 PFUs were plated. However, the total PFUs plated varied considerably between samples, from as few as 50,000 to as many as 700,000 PFUs. At both of these extremes, however, at least 15 mutants were identified and isolated.

The main and interacting effects of microenvironment, dose, and time after exposure on mutational frequency are shown in Figure 3. This statistical model was developed by assuming a linear relationship between the variables and mutational frequency. Significant changes in mutational frequency were found when comparing the main effects of microenvironment (3D vs $2 \mathrm{D})(P<0.01)$, dose $(P<0.05)$, and time $(P<$ 0.05). A significant difference was also noted for the interaction parameter dose and time $(P<0.05)$.

The main effect of dose and the main effect of time of culture after exposure on mutational frequency were also analyzed as nominal factors to examine their linear 
relationship. This evaluation allowed analysis of microenvironment and dose (Figure 4) assuming a linear relationship between culture time after exposure and mutational frequency. That assumption (a linear response of culture time after exposure) was tested by assuming it to be a nominal factor and verified by the linear shape of the curve (Figure 5).

\subsection{Packaging Efficiency}

The relationship between packaging efficiency (normalized to unirradiated control samples) and mutational frequency was also evaluated (Figure 6). Qualitatively, the packaging efficiency decreased with increasing mutational frequency. Also, packaging efficiency seemed to be higher for cells cultured in 2D monolayers $(17,800 \pm 8,300$ $\mathrm{PFU} / \mu \mathrm{g}$ DNA) than for the $3 \mathrm{D}$ samples $(7,600 \pm 4,900 \mathrm{PFU} / \mu \mathrm{g} \mathrm{DNA})$.

\subsection{Mutational Spectrum}

Approximately 300 mutations were found within the LacI region of all cells tested. The mutations unique to the irradiated samples (i.e., excluding mutations found in the un-irradiated controls) are shown in Table 1. The irradiated samples showed 18 unique deletions ranging from 1 to $662 \mathrm{bp}$ in length. Seven samples contained multiple mutations in the same target.

Analysis of unique mutations grouped by microenvironment, dose, and time of culture revealed no statistical differences (Figure 7). Although no statistical differences were found between tested groups, the proportion of mutations that were deletions was found to increase with increasing dose. 


\section{Discussion}

The lacI transgene used in this study proved to be a sensitive target for assessing HZE ionizing radiation damage, capable of identifying not only point mutations but also relatively large deletions ( $>660 \mathrm{bp}$ ) as well as insertions. However, only 1 of 22 unique deletions, or fewer than $2 \%$ of the total unique mutations identified, were longer than 600 $\mathrm{bp}$, suggesting that analysis of a still smaller target might be able to capture most of the mutational events in this size range. The late-stage biological impact of deletions of this or much larger size relative to the impact of a missense or nonsense mutation are still unclear.

Although this model used the 1,100 -bp LacI target to quantitatively assess DNA mutagenesis, a correlation was found between mutational frequency and packaging efficiency. Assessment of packaging efficiency, which makes use of the entire $45.5-\mathrm{kbp}$ lambda DNA, might prove to be a potential method of identifying much larger deletions and DNA damage, both of which have been demonstrated in both transgenic and native targets (Chang et al., 2001).

The combined findings regarding mutational frequency and packaging efficiency suggest that the culture microenvironment has significant influence on mutagenesis in a cell. Cells and cell aggregates cultured in the bioreactor experience signals in a 3D microenvironment that is drastically different from that of cells cultured in the 2D tissueculture flasks and presumably more akin to the in vivo condition. 
An increasing mutational frequency was also evident with continued culture after exposure to HZE radiation. Although increases such as this have been reported elsewhere (Zimmer et al., 1996, Winegar et al., 1994), the mutational frequency usually tends to level off within a shorter time than that analyzed here. This suggests that the normal DNA repair processes may have been impaired with the type of mutations and DNA damage caused by HZE radiation.

This study also demonstrated significant interactions among the factors examined. For example, samples exposed to low radiation doses showed less of an increase in mutational frequency over time than did those exposed to higher doses. In other words, the greater the damage initially received, the more that damage was amplified with increasing culture time.

\section{Summary}

Studies using transgenic models are providing significant amounts of information on the frequency and type of mutations induced by specific mutagens at the DNA level. However, the fact that many such studies are using 2D models to elucidate some of the uncertainties in HZE-induced mutagenesis could introduce another bias-that of the 2D culture system. Our results suggest that a 3D tissue-equivalent model may be better for attempting to elucidate human in vivo conditions when using an in vitro model system. 


\section{Acknowledgments}

The authors acknowledge Dr. Alan Feiveson for his assistance in statistical evaluation and Brookhaven National Laboratory for providing the facilities that made this study possible. Funding was provided by National Aeronautics and Space Administration NRA 101-31-GNDA.

\section{References}

Balmain, A. and Harris, C. C. (2000) Carcinogenesis, 21, 371-7.

Barcellos-Hoff, M. H. and Brooks, A. L. (2001) Radiat Res, 156, 618-27.

Cairns, J. (2002) Proc Natl Acad Sci U S A, 99, 10567-70.

Chang, P. Y., Kanazawa, N., Lutze-Mann, L. and Winegar, R. (2001) Phys Med, 17 Suppl 1, 189-91.

Gonda, S. R., Wu, H., Pingerelli, P. L. and Glickman, B. W. (2001) Adv Space Res, 27, 421-30.

Grosovsky, A., Bethel, H., Parks, K., Ritter, L., Giver, C., Gauny, S., Wiese, C. and Kronenberg, A. (2001) Phys Med, 17 Suppl 1, 238-40.

Ikehata, H., Masuda, T., Sakata, H. and Ono, T. (2003) Environ Mol Mutagen, 41, $280-$ 92.

Kohler, S. W., Provost, G. S., Fieck, A., Kretz, P. L., Bullock, W. O., Sorge, J. A., Putman, D. L. and Short, J. M. (1991) Proc Natl Acad Sci U S A, 88, 7958-62.

Kronenberg, A. (1994) Adv Space Res, 14, 339-46. 
Kronenberg, A., Gauny, S., Criddle, K., Vannais, D., Ueno, A., Kraemer, S. and Waldren, C. A. (1995) Radiat Environ Biophys, 34, 73-8.

Kronenberg, A. and Little, J. B. (1989) Int J Radiat Biol, 55, 913-24.

Lyon, J. G. (2000) Science, 288, 1987-91.

Masumura, K., Kuniya, K., Kurobe, T., Fukuoka, M., Yatagai, F. and Nohmi, T. (2002) Environ Mol Mutagen, 40, 207-15.

McKinzie, P. B., Delongchamp, R. R., Heflich, R. H. and Parsons, B. L. (2001) Mutat Res, 489, 47-78.

Mirsalis, J. C. (1995) Toxicol Lett, 82-83, 131-4.

Nohmi, T., Suzuki, T. and Masumura, K. (2000) Mutat Res, 455, 191-215.

Pei, M., Solchaga, L. A., Seidel, J., Zeng, L., Vunjak-Novakovic, G., Caplan, A. I. and Freed, L. E. (2002) Faseb J, 16, 1691-4.

Provost, G. S., Kretz, P. L., Hamner, R. T., Matthews, C. D., Rogers, B. J., Lundberg, K. S., Dycaico, M. J. and Short, J. M. (1993) Mutat Res, 288, 133-49.

Rogers, B. J., Provost, G. S., Young, R. R., Putman, D. L. and Short, J. M. (1995) Mutat Res, 327, 57-66.

Setlow, R. B. (1999) Mutat Res, 430, 169-75.

Vogel, E. W., Nivard, M. J., Ballering, L. A., Bartsch, H., Barbin, A., Nair, J., Comendador, M. A., Sierra, L. M., Aguirrezabalaga, I., Tosal, L., Ehrenberg, L., Fuchs, R. P., Janel-Bintz, R., Maenhaut-Michel, G., Montesano, R., Hall, J., Kang, H., Miele, M., Thomale, J., Bender, K., Engelbergs, J. and Rajewsky, M. F. (1996) Mutat Res, 353, 177-218. 
Winegar, R. A., Lutze, L. H., Hamer, J. D., O'Loughlin, K. G. and Mirsalis, J. C. (1994) Mutat Res, 307, 479-87.

Zhang, J., Campbell, R. E., Ting, A. Y. and Tsien, R. Y. (2002) Nat Rev Mol Cell Biol, 3, 906-18.

Zimmer, D. M., Zhang, X. B., Harbach, P. R., Mayo, J. K. and Aaron, C. S. (1996) Environ Mol Mutagen, 28, 325-33. 
Table 1. Unique mutations in 2D and 3D samples exposed to $1 \mathrm{GeV} / \mathrm{amu}$ iron charged particles.

\begin{tabular}{|c|c|c|c|c|c|c|c|c|}
\hline \multirow[b]{2}{*}{ Type } & \multirow[b]{2}{*}{ Dose (Gy) } & \multirow[b]{2}{*}{ Time (Days) } & \multicolumn{3}{|c|}{ 3D } & \multicolumn{3}{|c|}{$2 D$} \\
\hline & & & Position & From & To & Position & From & To \\
\hline \multirow[t]{36}{*}{ Transversions } & 0.1 & 0 & 833 & $\mathrm{C}$ & A & 104 & c & A \\
\hline & & & 886 & A & C & & & \\
\hline & 0.1 & 7 & 109 & $\mathrm{C}$ & A & 784 & G & $\mathrm{C}$ \\
\hline & & & 169 & $\mathrm{C}$ & A & & & \\
\hline & 0.25 & 0 & 170 & A & $\mathrm{T}$ & 66 & $\mathrm{C}$ & $\mathrm{G}$ \\
\hline & & & 618 & G & $\mathrm{C}$ & 350 & A & $\mathrm{T}$ \\
\hline & & & 781 & G & $\mathrm{T}$ & 861 & $\mathrm{C}$ & A \\
\hline & & & & & & 862 & A & $\mathrm{C}$ \\
\hline & & & & & & 1007 & G & $\mathrm{C}$ \\
\hline & 0.25 & 1 & & & & 121 & C & G \\
\hline & & & & & & 431 & $G$ & $\mathrm{~T}$ \\
\hline & 0.25 & 7 & & & & 259 & C & A \\
\hline & 0.5 & 0 & 861 & C & A & 527 & $\mathrm{C}$ & A \\
\hline & & & 862 & A & $\mathrm{C}$ & & & \\
\hline & 0.5 & 1 & 274 & $\mathrm{C}$ & A & 140 & G & $\mathrm{C}$ \\
\hline & & & 486 & G & $\mathrm{C}$ & 776. & $\mathrm{C}$ & A \\
\hline & & & 1094 & $\mathrm{C}$ & A & & & \\
\hline & & & 1096 & A & $\mathrm{C}$ & & & \\
\hline & & & 1097 & $\mathrm{C}$ & G & & & \\
\hline & 0.5 & 7 & 197 & G & C & 528 & C & A \\
\hline & & & 856 & G & $T$ & & & \\
\hline & 1 & 0 & 56 & G & C & 166 & $T$ & G \\
\hline & 1 & 1 & 79 & $T$ & A & 133 & G & $\mathrm{C}$ \\
\hline & 2 & 0 & 180 & G & C & 169 & $\mathrm{C}$ & A \\
\hline & 2 & 0 & 194 & $\mathrm{C}$ & G & 189 & A & $\mathrm{T}$ \\
\hline & 2 & 0 & 350 & A & $\mathrm{T}$ & 582 & $\mathrm{~T}$ & A \\
\hline & & & 784 & G & $\mathrm{C}$ & & & \\
\hline & & & 832 & $\mathrm{C}$ & A & & & \\
\hline & & & 1010 & A & $\mathrm{C}$ & & & \\
\hline & 2 & 1 & 56 & G & C & 384 & $T$ & A \\
\hline & & & 190 & A & C & & & \\
\hline & & & 273 & $\mathrm{C}$ & A & & & \\
\hline & & & 558 & $\mathrm{~T}$ & A & & & \\
\hline & & & 830 & $\mathrm{~T}$ & A & & & \\
\hline & 2 & 7 & 162 & $\mathrm{~T}$ & G & 167 & $\mathrm{~T}$ & $\mathrm{G}$ \\
\hline & & & 873 & $T$ & A & & & \\
\hline \multirow[t]{3}{*}{ Transitions } & 0.1 & 0 & & & & 294 & C. & $T$ \\
\hline & & & & & & 782 & G & A \\
\hline & 0.1 & 1 & 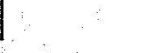 & & & 132 & C & $T$ \\
\hline
\end{tabular}




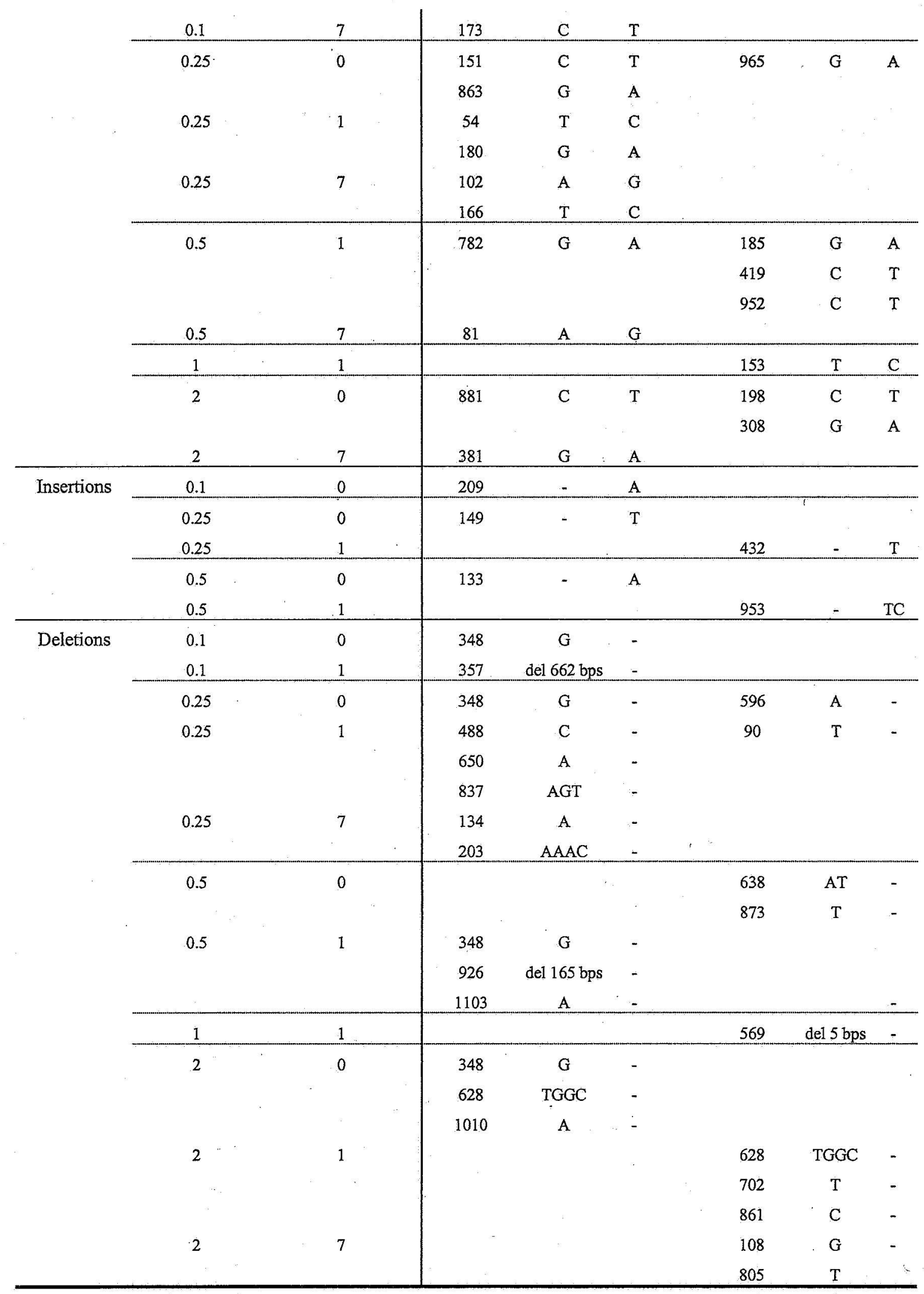




\section{Figure Legends}

Figure 1. Changes in glucose (squares) and lactate (triangles) levels during the formation of the 3D aggregates representing the tissue-equivalent samples. Measurements were taken before (open) and after (filled) introduction of fresh medium into each of 8 bioreactors. Error bars indicate standard error of the model.

Figure 2. (A) Mutational frequency and (B) normalized packaging efficiency of Rat2 $\lambda$ cells in 2D cell culture exposed to various concentrations of the mutagen ethylnitrosourea (ENU).

Figure 3. Main and interacting effects of microenvironment, dose, and time after exposure on mutational frequency. Microenvironment is defined as difference in samples cultured in $3 \mathrm{D}$ and 2D. Asterisks represent significant changes in mutational frequency between the levels tested.

Figure 4. Main effects of (A) microenvironment and (B) dose after irradiation on mutational frequency. The mutational frequency intercept, representing the background frequency level, of the model was $6.97 \times 10^{-5}$.

Figure 5. Main effect of culture time after irradiation on mutational frequency.

Figure 6. Relationships between packaging efficiency and mutational frequency. Closed diamonds represent 2D monolayer cultures; open squares are the 3D aggregates. 
Figure 7. Relationship between types of unique mutations [transversions (TV), transitions (TS), insertions (INS), and deletions (DEL)] grouped by (A) microenvironment, (B) dose, and (C) time. 


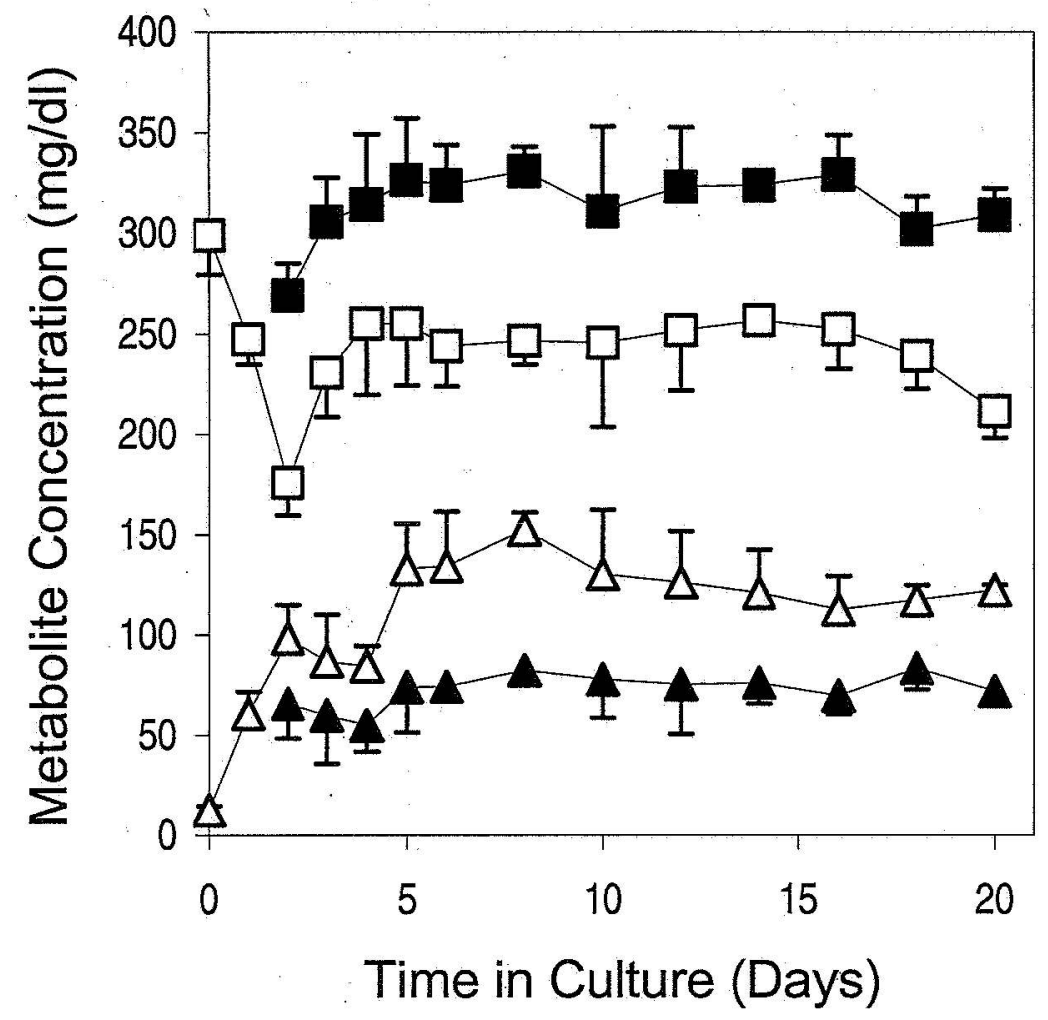

fig 1 


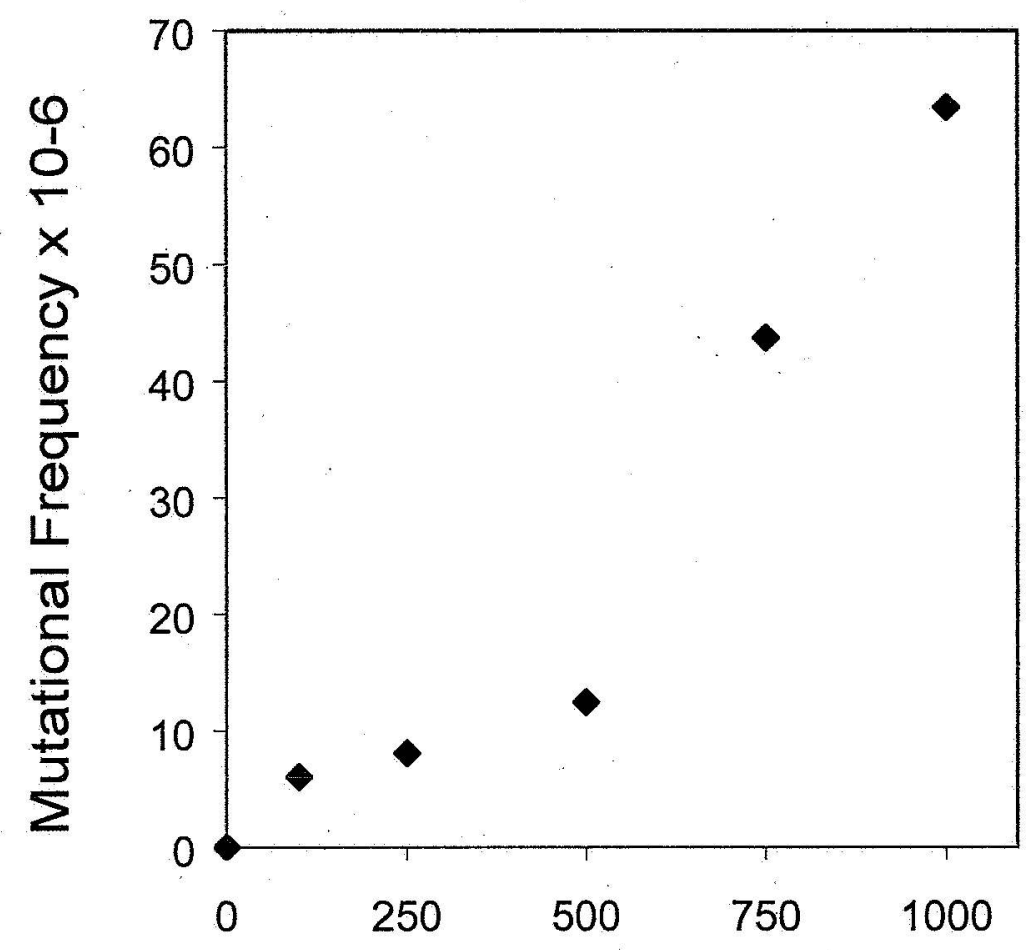

(A) ENU Concentration (ug/ml)

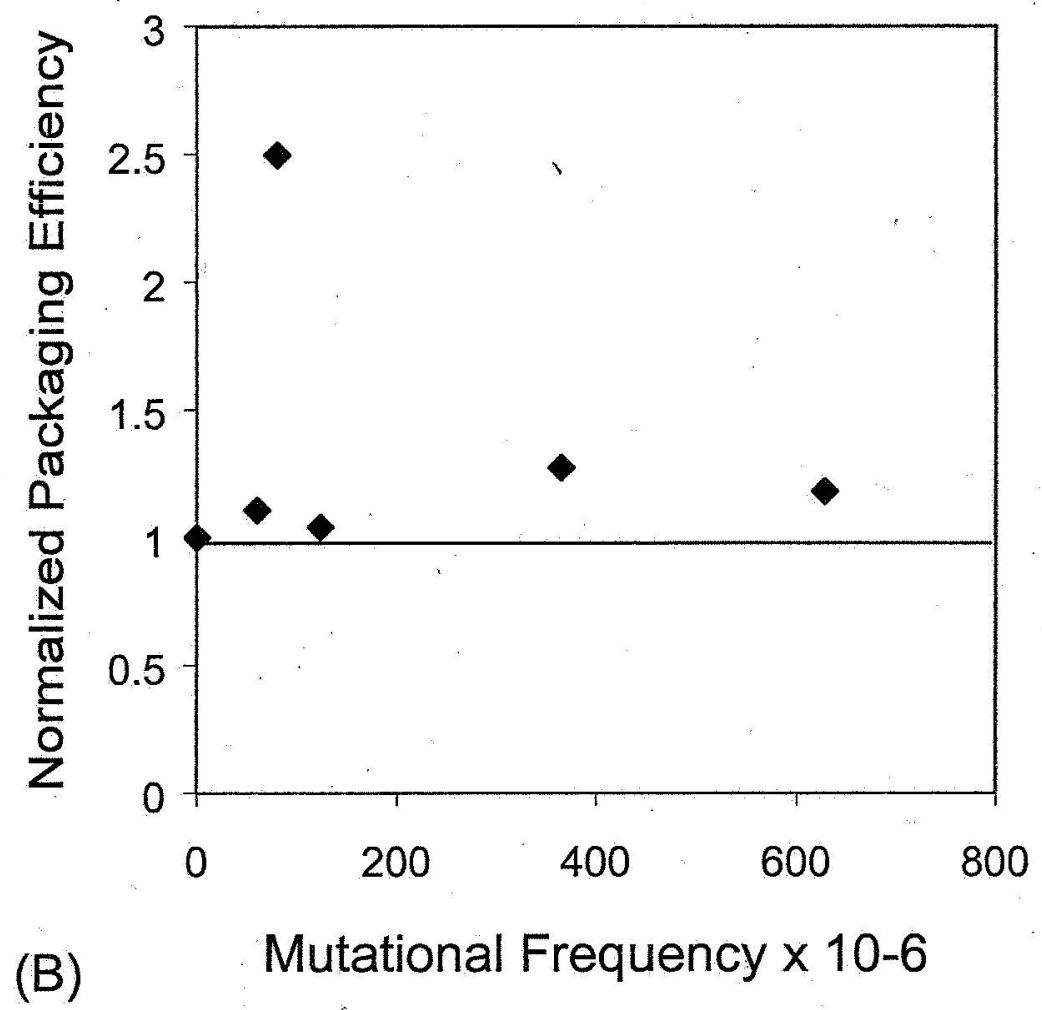

Fig 2 


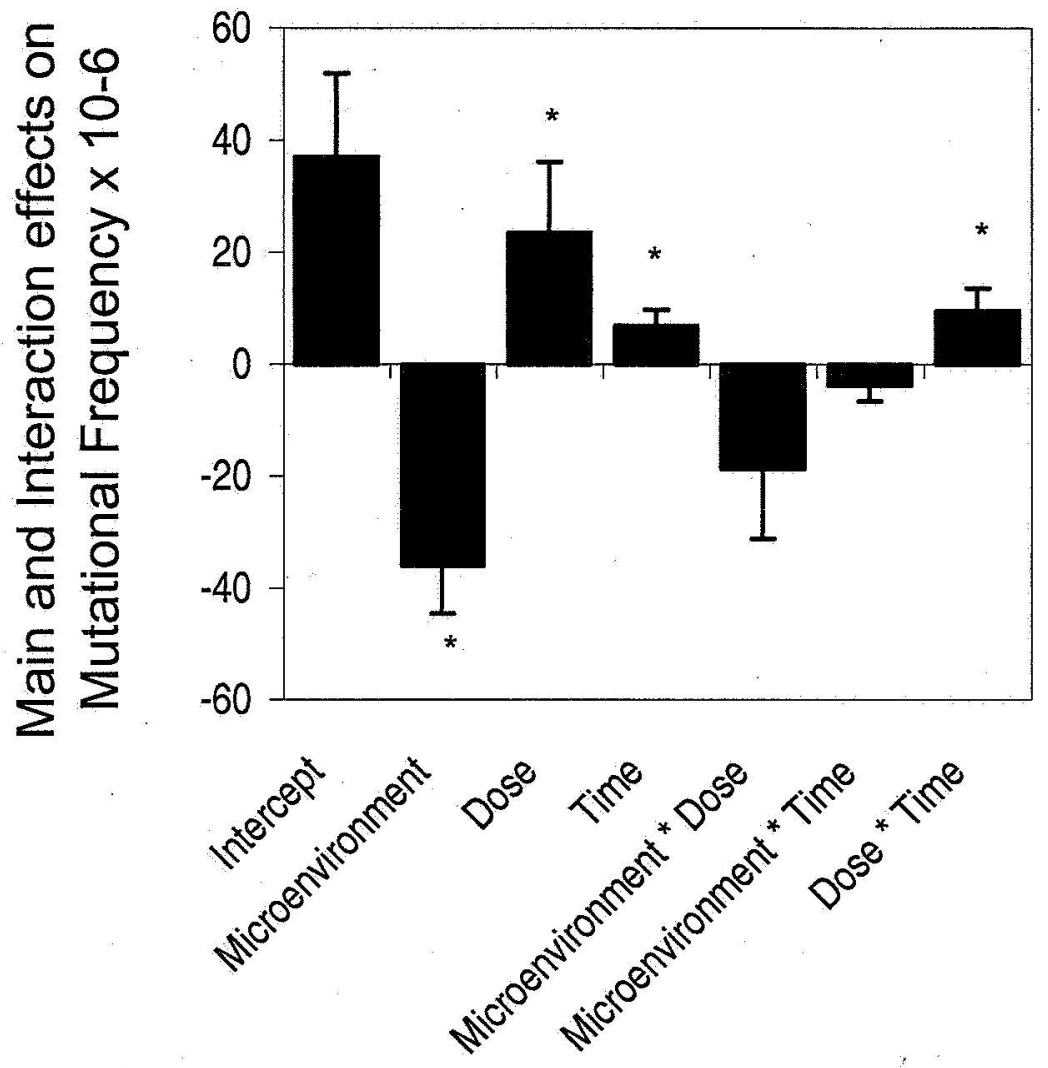

fig 3 


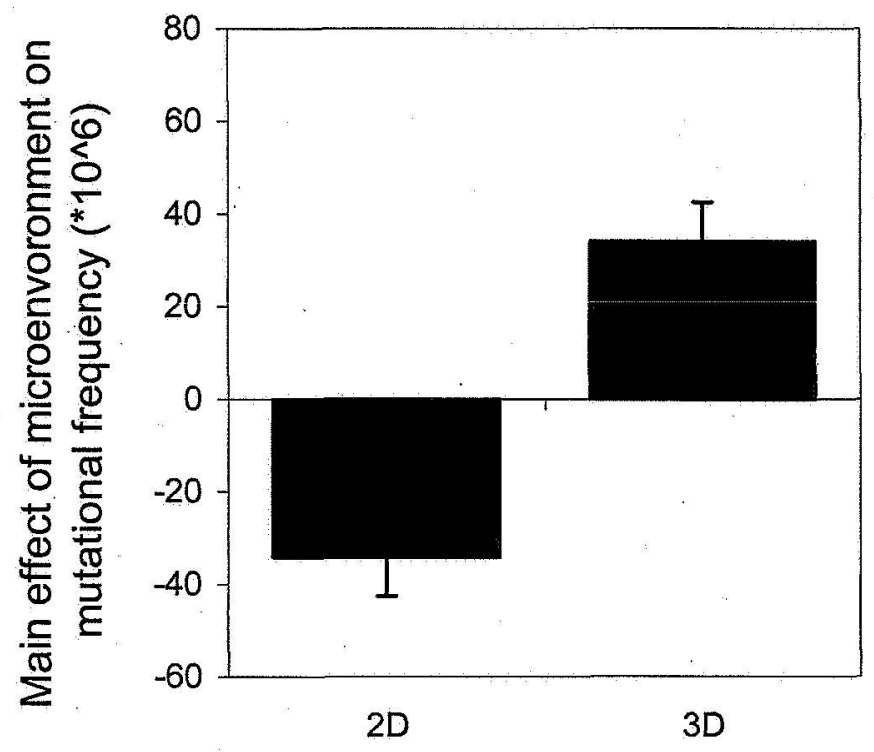

(A)

Microenvironment

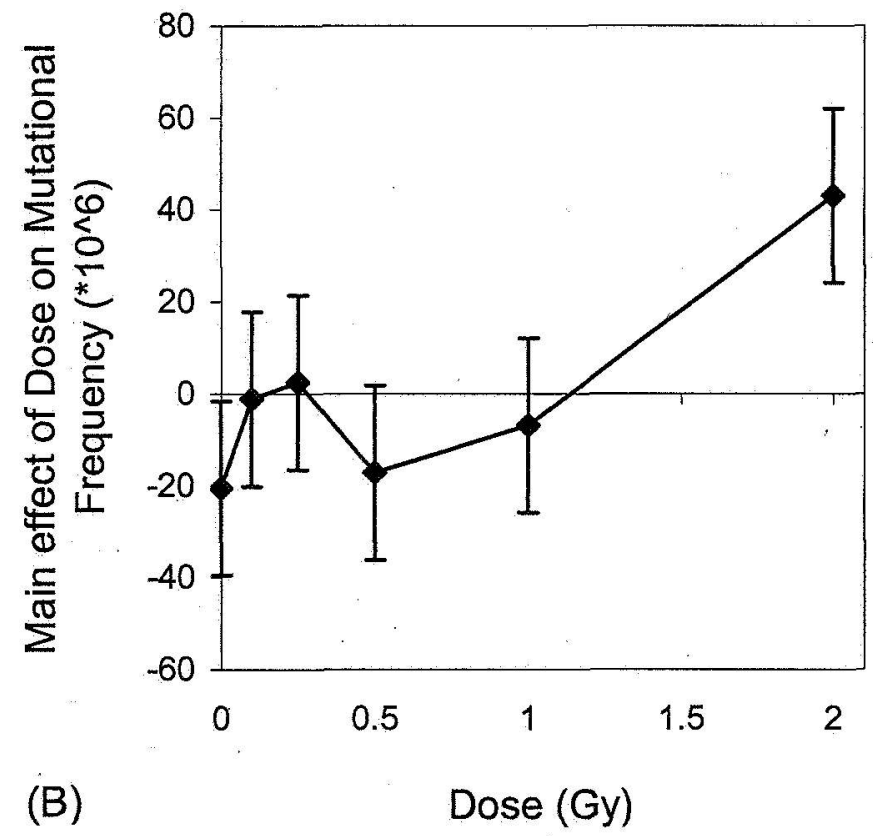

fig 4 


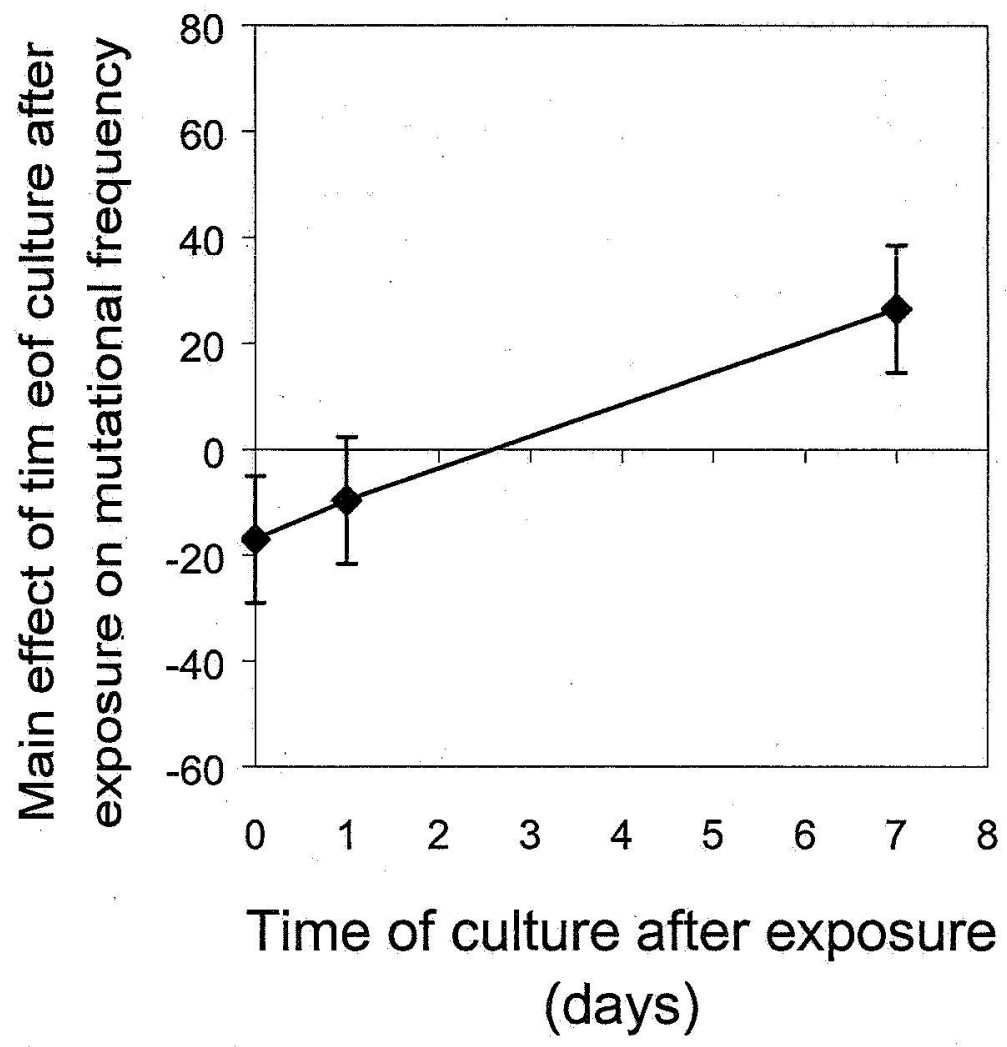

Figure 5 


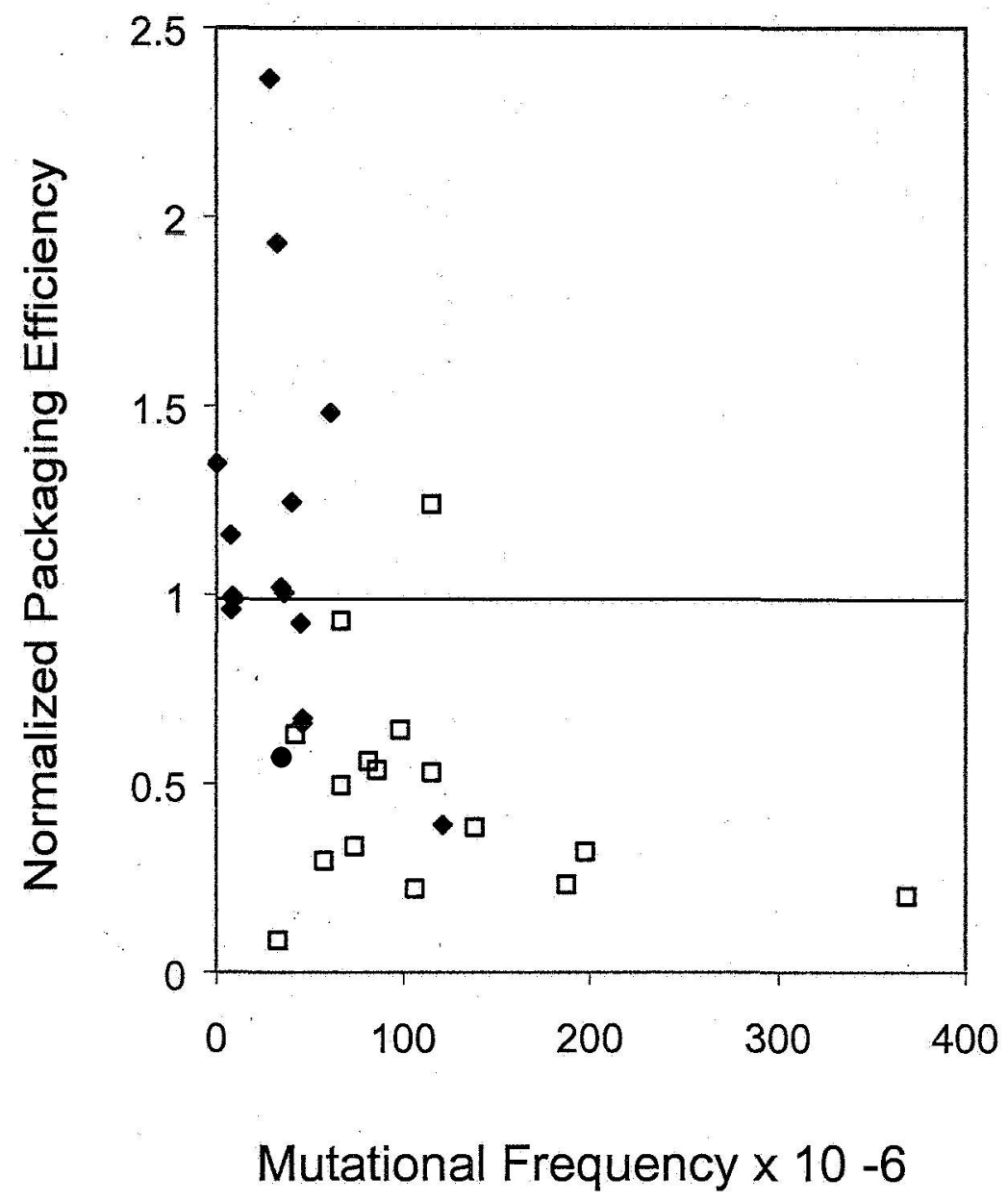

fig 6 


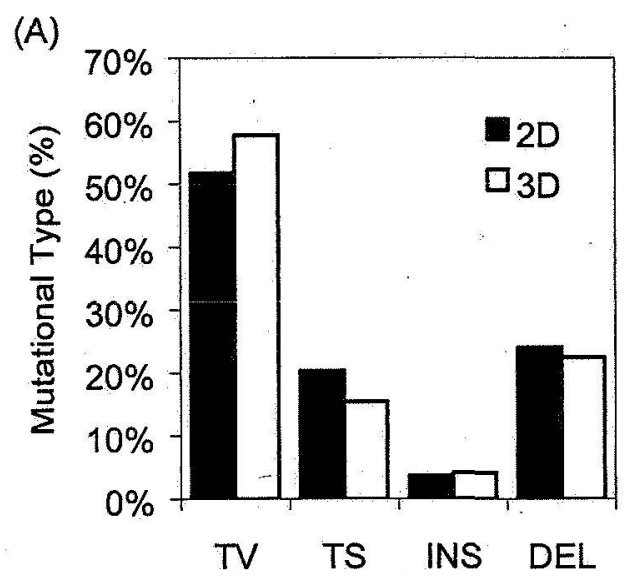

(B)
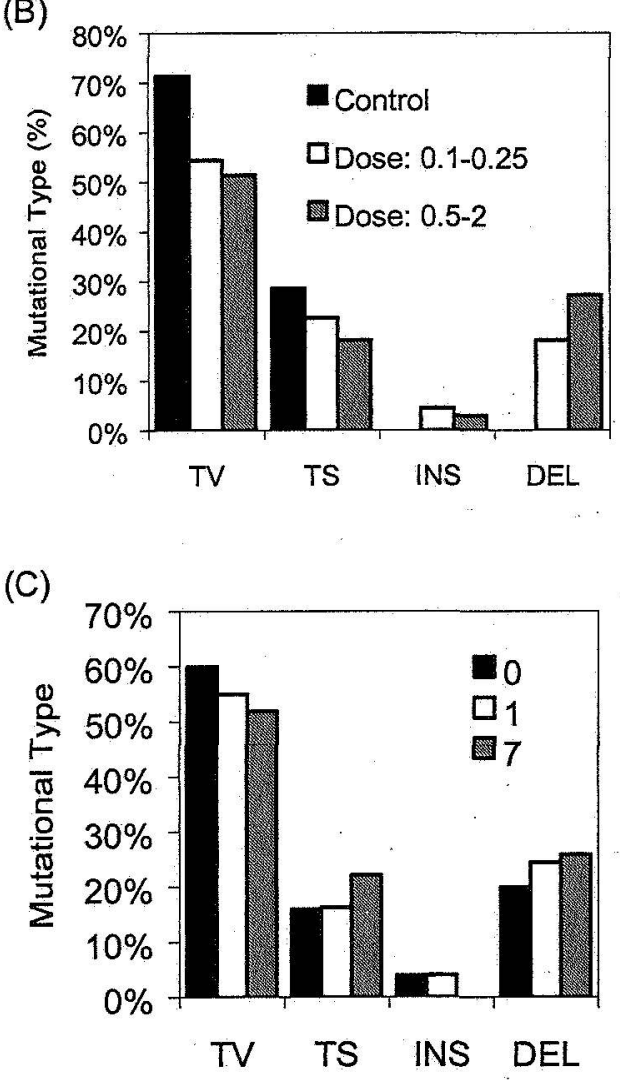

Fig 7 